\title{
COMPOUND DRY-HOT EXTREME EVENTS IMPROVING INDIVIDUAL AND COMMUNITY PREPAREDNESS AND RESPONSE
}

\section{KEY MESSAGES}

- Need for improved individual and community preparedness and response to compound dry-hot extreme events.

- Compound dry-hot extreme events action plans should address both current and future needs.

- Bottom-up approaches should be used to develop and implement compound dry-hot extreme events action plans.

- Call for action to understand how compound dry-hot extreme events action plans can look like.

- Ongoing conversations need to be had about how to protect and promote health and well-being for all, everywhere.

\section{ABSTRACT}

The frequency of simultaneous or consecutive heatwaves, droughts and wildfires is increasing. Compound dry-hot extreme events, defined as a mix of concurrent or sequential occurrence of heatwaves, droughts and fires, contribute to risks and impacts to both society and the environment. Compound dry-hot extreme events already pose serious impacts on human health and well-being, and these impacts are expected to increase in the future. The purpose of this report is to discuss the need for improved individual and community preparedness and response to compound dry-hot extreme events, and to highlight the benefits such improvements would bring. In particular, the development and implementation of compound dry-hot extreme events action plans is essential for reducing the adverse health effects of current and future compound dryhot extreme events.

\section{INTRODUCTION}

The frequency of simultaneous heatwaves, droughts and fires is increasing (IPCC, 2021). Compound dryhot extreme events are defined as a mix of concurrent or sequential occurrence of heatwaves, droughts, and wildfires (IPCC, 2021; AghaKouchak et al., 2020; Papalexiou et al., 2018) which contribute to risks and impacts to society and the environment (Jay et al., 2021; Ebi et al., 2021). The purpose of this report is to discuss the need for improved individual and community preparedness and response to compound dry-hot extreme events. The background section contains information on the occurrence and impacts of these events and explains the need to develop and implement compound dry-hot extreme events action plans. The discussion section outlines the need for improved individual and community preparedness and responses and explains a few considerations that must be addressed. The conclusion tackles how individuals and communities can address compound dry-hot extreme events to protect and promote health and well-being. The recommendations include what needs to be done to improve preparedness and response to compound dry-hot extreme events to minimise impacts.

\section{Authors:}

| Nunes, Dr Ana Raquel, Warwick Medical School

University of Warwick 


\section{BACKGROUND}

Global climate change affects us all, but some more than others: it acts as a health risk amplifier and increases inequalities (Jay et al., 2021; Ebi et al., 2021; Nunes, 2021). The impacts of extreme events depend on the vulnerability, resilience and adaptation of the individuals and communities affected (Nunes, 2021). According to the IPPC's Working Group I, 6th Assessment Report, "Compound extreme events are the combination of multiple drivers and/or hazards that contribute to societal or environmental risk. Examples are concurrent heatwaves, droughts and compound fire weather conditions [...] or concurrent extremes at different locations" (IPCC, 2021: SPM11). Additionally, there is high confidence in the IPCC (2021) that "Concurrent heat waves and droughts have become more frequent over the last century, and this trend will continue with higher global warming". If we consider that individually occurring extreme events already produce serious impacts, we can only expect compound extreme events to be associated with more damaging effects (Sutanto et al., 2020; AghaKouchak et al., 2020; Papalexiou et al., 2018). Additionally, according to the principles of the Sendai Framework, effective disaster risk reduction depends on the mapping of risks and risk-informed strategies for both the prevention of disasters, and reduction of impacts when these occur (United Nations, 2015). Finally, it is crucial that we know who the most vulnerable are, and where, when, and how they are most vulnerable (Nunes, 2021, 2020a, 2020b, 2018, 2016).

With climate change, climate extremes and compound extreme events are expected to increase in frequency, intensity, and duration (IPCC, 2021). We are already experiencing increasing compounding and interconnected events, or compound extreme events. These can include extreme events that occur concurrently or sequentially, extreme events with underlying roots that magnify impact, or combined events that, although not extremes, contribute to an extreme event or impact (Zscheischler et al., 2018). Compound dry-hot extreme events can occur in a variety of patterns, as shown in Table 1.

Table 1: Patterns of compound dry-hot extreme events

\begin{tabular}{c|c}
$\begin{array}{c}\text { Extreme event } \\
\text { Drought }+ \\
\text { Heatwave }\end{array}$ & $\begin{array}{c}\text { Type of extreme event } \\
\text { Coment }\end{array}$ \\
\hline $\begin{array}{c}\text { Heatwave }+ \\
\text { Wildfire }\end{array}$ & $\begin{array}{c}\text { Compound dry-hot extreme } \\
\text { event }\end{array}$ \\
\hline $\begin{array}{c}\text { Drought }+ \\
\text { Wildfire }\end{array}$ & $\begin{array}{c}\text { Compound dry-hot extreme } \\
\text { event }\end{array}$ \\
\hline $\begin{array}{c}\text { Drought }+ \\
\text { Heatwave }+ \\
\text { Wildfire }\end{array}$ & $\begin{array}{c}\text { Compound dry-hot extreme } \\
\text { event }\end{array}$ \\
\hline
\end{tabular}

\section{IMPACTS AND DRIVERS}

Compound extreme events threaten human health and natural systems (AghaKouchak et al., 2020). The increased frequency, intensity and duration of extreme events and compound dry-hot extreme events pose risks and impacts to human health and well-being that can range from illness to death (IPCC, 2012).

We anticipate the impacts of such events to become more severe due to the increased exposure and vulnerability of urban populations and settings (AghaKouchak et al., 2020; Papalexiou et al., 2018). The inhabitants and infrastructure of large cities and megacities are disproportionally more affected (AghaKouchak et al., 2020; Papalexiou et al., 2018). Despite this, some regions of the world are more prone to certain extreme events, and the distribution of impacts is also unequal (Phillips et al., 2020; Sutanto et al., 2020; Vitolo et al., 2019). As a result, there is a need to improve our understanding of how to build resilience to compound extreme events (AghaKouchak et al., 2020), as we still need to do for extreme events (Nunes, 2020a).

It is vital to reduce the impacts of compound dry-hot extreme events, namely heatwaves, droughts and wildfires, as the impact from each individual hazard will be higher if there is an occurrence of compound dry-hot extreme events (Sutanto et al., 2020). Despite the high impacts from compound dry-hot extreme events, so far, most research has focused on single events rather than compound events (AghaKouchak et al., 2020; Zscheischler et al., 2018).

Nevertheless, and according to research by Sutanto and colleagues, drought triggers and increases the probability of heatwave, and vice versa, heatwave triggers and increases the probability of drought occurring. On the other hand, drought increases the probability of ignition and fires, but fire does not trigger or increase the probability of drought. Heatwave increases the probability of fire, and fire triggers and increases the probability of heatwave (Sutanto et al., 2020).

\section{VULNERABILITY, RESILIENCE AND ADAPTATION}

Protecting and promoting human health and well-being is imperative as almost all impacts are preventable (e.g., Jay et al., 2021; Ebi et al., 2021). If no action is taken to reduce these impacts, we will continue to see more extreme events occurring with high loss of human lives (IPCC, 2021; IPCC, 2012).

Understanding the root causes of vulnerability, the drivers of resilience and what shapes adaptation to extreme events has been the focus of research for a long time (Nunes, 2021, 2020a, 2020b, 2018, 2016). Assets have been found to be a key determinant of vulnerability, resilience and adaptation. 
Vulnerability was found not to be a key determinant of resilience, which is mostly influenced by an understanding of the challenges posed by threats and feeling motivated to act, thus being a key determinant of adaptation (Nunes, 2021). Most recently, this interest has also turned to understanding vulnerability, resilience and adaptation to compound dry-hot extreme events following the last IPCC Working Group I report (IPCC, 2021).

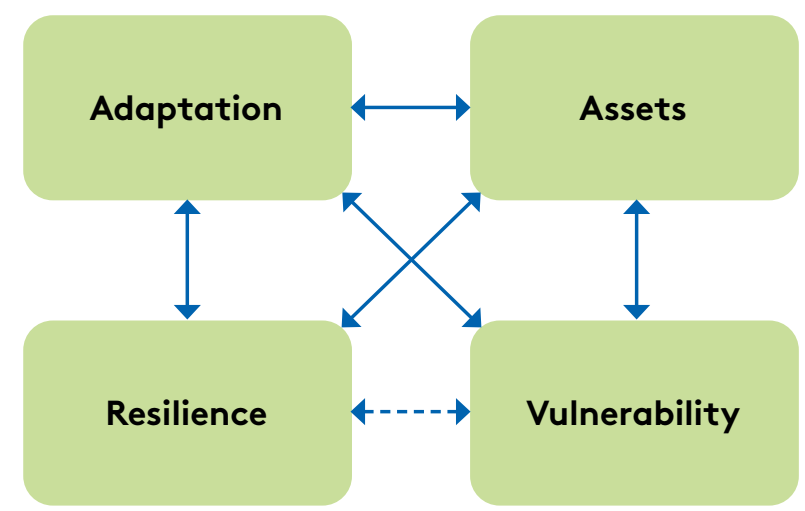

Figure 1. Relationship between assets, vulnerability, resilience and adaptation. (Source: Nunes, 2021)

Legend: Straight arrow represents being a key determinant and dotted arrow represents not being a key determinant.

\section{PREPAREDNESS AND RESPONSE}

As we move forward, we must also think about how we manage heatwaves, droughts and wildfires when they occur concurrently or sequentially, as the overall impacts are expected to be higher than the sum of each events' impacts. Many countries have thus developed and implemented heatwave action plans, drought action plans and wildfires action plans. Such plans aim to improve preparedness and response to such events, are usually updated annually and used separately by service providers, despite such hazards may occur concurrently or sequentially - compound dry-hot extreme events.

Because some places experience these events simultaneous there is a call for action plans to focus on a combination of hazards considering the current and future vulnerability of people and places (Kruczkiewicz et al., 2021). Despite this, we are still using one-hazard approaches to these problems which are most often siloed, disjointed and scant instead of a multi-hazard approach (Kruczkiewicz et al., 2021) to reduce vulnerability, build resilience and improve adaptation of people and places. Building resilience can have numerous outcomes which can include long-term sustainability transformations (Nunes, 2021, 2020; Cutter, 2020). Two-way discussions among different stakeholders are vital for effective risk communication, build resilience and improve adaptation (Kruczkiewicz et al 2021).

If we are to plan for better preparedness and response to compound dry-hot extreme events we need to use a whole-system approach and a health-in-all policies approach (Leppo et al., 2013), addressing micro-, meso- and macro-level benefits and trade-offs of actions and strategies developed and implemented, so we are better prepared to promote and protect health and well-being. To address this, the challenge is to rethink compound extreme events risks in order to build sustainable and resilient urban settings and communities. As with heatwaves, droughts and wildfires are an all-off-society problem that impact on all sectors and all levels with wide ranging exacerbation of risks and requiring wide range of actions.

\section{ACTION PLANS}

The aim of compound dry-hot extreme events action plans must be to protect human lives from the dangers and impacts of heatwaves, droughts and wildfires, providing preparedness and response advice and guidance on how to protect and promote health and well-being, and reduce harm and negative impacts. Such action plans, as with individual extreme events action plans (e.g., Heatwave Plan), need to include a series of suggested actions that decisionmakers, commissioners of services, service providers, organisations and institutions, communities and individuals at local and national levels can easily use and implement to minimise impacts. These action plans should also include risk communication and public-facing resources, so their reach can be expanded to all those who need to access that information.

If the above are addressed, a question persists on how we can progress from developing and implementing compound dry-hot extreme events action plans, to consistently reducing impacts on vulnerable people and places. The all-of-society and whole-of-society approaches are one answer, but anticipatory action, participatory action, and risk communication are also needed. To ensure the protection and promotion of human health, these actions need to be interwoven with bottom-up approaches which involve the most vulnerable and worst affected by the impacts of compound dry-hot extreme events.

\section{DISCUSSION}

As we know, compound dry-hot extreme events are already occurring and are projected to occur more frequently, more intensely and for longer periods (IPCC, 2021, 2012). We need to take holistic, all-of society, whole-of-society approaches to how we prepare and respond to them now and consider how we want to prepare and respond in the future. 
The current focus of action plans on emergency preparedness and response must shift and expand: an extreme events action plan with a whole-system approach outlook must have short-, medium- and longer-term adaptation as its core aim. To achieve this, partnerships are essential. A health-in-all policies approach at the core (Leppo et al., 2013) of those partnerships will help establish health as both a precondition and an outcome of policies and actions that are implemented in other sectors of society (Nunes, et al., 2016). This requires working with sectors outside of health, such as housing, transport, energy, business, economy, infrastructure, and so forth (Nunes, et al., 2016).

It is high time we discussed the need for better preparedness and response, and called for plans that aim to both reduce vulnerability, build resilience and improve adaptation. This can be achieved by focusing on action plans already in place, which can be implemented before and during a compound dry-hot extreme event to reduce its negative impacts, drawing on worldwide experience, learning and best practice. A good compound extreme event action plan should address what individuals and communities need to better prepare and respond to such events. This in turn requires a deeper understanding of the drivers and sources of vulnerability, resilience and adaptation; the impacts of compound dry-hot extreme events on human health and well-being; the means of protecting and promoting human health and well-being.

The importance of compound dry-hot extreme event action plans and early warning systems needs to be highlighted if we are to improve our preparedness and response. This can be achieved through exploring and mapping compound dry-hot extreme events, to reduce vulnerability and increase the resilience of people and places for better adaptation and reduced impacts.

All these actions aim at putting into context and provide a view for the identification of hotspots and the assessment of compound extreme event patterns that can be used to determine the interrelationship of extreme events.

It is about understanding compound risks and complex emergencies, and the need for novel approaches to preparedness and response. As well as make it clear that a multi-hazards approach can help us reduce risk from compound dry-hot extreme events, improve the way we communicate about compound risks, and bring the whole of society to the table.

\section{CONCLUSION}

The need for improved individual and community preparedness and response to compound dry-hot extreme events cannot be ignored. Individual and community preparedness and response to compound dry-hot extreme events have many short-, mediumand long-term benefits. They have the potential to address a myriad of climate change issues. Many countries are starting to develop and implement compound dry-hot extreme events action plans to address issues of risks and impacts. Countries and cities will benefit from better partnerships between national and local government, and across all sectors of society (e.g., health and social care, transport, housing, energy, business). Individuals and communities will benefit from better ways and actions to prepare, respond, and reduce impacts. And all will benefit from better planning, coordination, communication, preparedness and response. This will lead to better management of compound dry-hot extreme events that leads to reduction of impacts.

Compound dry-hot extreme events action plans are a short-, medium- and long-term investment in health and well-being. It is crucial to adopt an all-of-society approach which involves individuals, community, institutions and policymakers to improve and support disaster planning, response and management.

Additionally, it is essential to prepare for different possible futures, among them a warmer future, and develop actions for the short-, medium- and longerterm to be implemented at all levels of society to make sure we are better prepared to respond.

\section{RECOMMENDATIONS}

Given the need for improved individual and community preparedness and response to compound dry-hot extreme events, all countries and cities should develop and implement compound dry-hot extreme events action plans that address both current needs and that account for different future needs.

An important first step is taking a bottom-up approach to develop and implement a participatory and clear all-of-society action plan on how individuals and communities are supported prior to and during a compound dry-hot extreme event.

A call to action to think and discuss how compound dry-hot extreme events action plans can look like, and an ongoing conversation about how to protect and promote health and well-being for all, everywhere. 


\section{REFERENCES}

1. AghaKouchak, A., F. Chiang, L.S. Huning, C.A. Love, I. Mallakpour, O. Mazdiyasni, H. Moftakhari, S.M. Papalexiou, E. Ragno, and M. Sadegh, 2020: Climate extremes and compound hazards in a warming world. Ann. Rev. Earth Planet. Sci., 48, no. 1, 519-548, https://doi.org/10.1146/annurev-earth-071719-055228

2. Cutter, S. (2020) Community resilience, natural hazards, and climate change: Is the present a prologue to the future?, Norsk Geografisk Tidsskrift - Norwegian Journal of Geography, 74:3, 200-208, https://doi.org/10 1080/00291951.2019.1692066

3. IPCC, 2021: Climate Change 2021: The Physical Science Basis. Contribution of Working Group I to the Sixth Assessment Report of the Intergovernmental Panel on Climate Change [Masson-Delmotte, V., P. Zhai, A. Pirani, S. L. Connors, C. Péan, S. Berger, N. Caud, Y. Chen, L. Goldfarb, M. I. Gomis, M. Huang, K. Leitzell, E. Lonnoy, J. B. R. Matthews, T. K. Maycock, T. Waterfield, O. Yelekçi, R. Yu and B. Zhou (eds.) ]. Cambridge University Press. In Press. www.ipcc.ch/report/arb/ wg1/downloads/report/IPCC_AR6_WGI_Full_Report.pdf (Last accessed: 20/10/2021)

4. IPCC, 2012: Managing the Risks of Extreme Events and Disasters to Advance Climate Change Adaptation. Cambridge University Press, 582 pp. www.ipcc.ch/ report/managing-the-risks-of-extreme-events-anddisasters-to-advance-climate-change-adaptation/ (Last accessed: 20/10/2021)

5. Kruczkiewicz, A., Klopp, J., Fisher, J. et al. (2021). Compound risks and complex emergencies require new approaches to preparedness. Proceedings of the National Academy of Sciences. 118. https://doi.org/10.1073/pnas.2106795118

6. Leppo K, Ollila E, Pena S, et al. Health in all policies: seizing opportunities, implementing policies. Helsinki: Ministry of Social Affairs and Health, Finland, 2013:1-358. www.euro.who.int/__data/assets/pdf_ file/0007/188809/Health-in-All-Policies-final.pdf (Last accessed: 20/10/2021)

7. Nunes, AR (2021) Exploring the interactions between vulnerability, resilience and adaptation to extreme temperatures. Nat Hazards. https://doi.org/10.1007/ s11069-021-04919-y
8. Nunes AR (2020a) Determinants of general and specified resilience to extreme temperatures. Weather Clim Soc 12 (4):913-928. https://doi.org/10.1175/ WCAS-D-19-0078.1

9. Nunes AR (2020b) General and specified vulnerability to extreme temperatures among older adults. Int J Environ Health Res 30 (5):515-532

10. Nunes AR (2018) The contribution of assets to adaptation to extreme temperatures among older adults. PLOS ONE 13:e0208121

11. Nunes AR (2016) Assets for health: linking vulnerability, resilience and adaptation to climate change. Tyndall Cent Clim Chang Res Work Paper 163:1-41

12. Nunes $A R$, Lee $K, O^{\prime}$ Riordan $T$ (2016). The importance of an integrating framework for achieving the Sustainable Development Goals: the example of health and wellbeing. BMJ Global Health, 1(3), pp. 1-12.

13. Papalexiou SM, AghaKouchak A, Trenberth KE, FoufoulaGeorgiou E (2018) Global, regional, and megacity trends in the highest temperature of the year: diagnostics and evidence for accelerating trends. Earths Future 6:71-78. https://doi.org/10.1002/2017EF000709

14. Poljansek, K., Marin Ferrer, M., De Groeve, T. and Clark, I., editor(s), Science for Disaster Risk Management 2017: Knowing better and losing less, Publications Office of the European Union, Luxembourg, 2017, ISBN 978-9279-60679-3 (main report online),978-92-79-60678-6 (main report print), 978-92-79-69673-2 (executive summary online),978-92-79-69674-9 (executive summary print),978-92-79-74167-8 (executive summary ePub), JRC102482.

15. United Nations, 2015. Sendai framework for disaster risk reduction 2015-2030. In: UN world conference on disaster risk reduction, 2015 March 14-18, Sendai, Japan. Geneva: United Nations Office for Disaster Risk Reduction; 2015

16. Zscheischler, J., Westra, S., van den Hurk, B.J.J.M. et al. Future climate risk from compound events. Nature Clim Change 8, 469-477 (2018). https://doi.org/10.1038/ s41558-018-0156-3 


\section{THE COP26 UNIVERSITIES NETWORK}

The COP26 Universities Network Climate Risk Notes are an outcome of the Climate Risk Summit. The Summit aimed to bring key information on the risks of climate change and their communication to decision-makers to the attention of a wider audience in advance of COP26. The Climate Risk Notes crystallise and expand on the learnings from the Summit. The Climate Risk Notes series is published in association with the UK COP26 Universities Network, however all opinions are those of the named authors rather than those of the network as a whole.

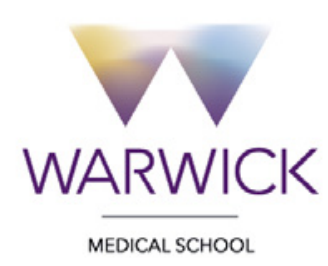

\section{HOW TO CITE THIS PAPER}

Nunes, A. R. (2021). Compound dry-hot extreme events improving individual and community preparedness and response. COP26 Universities Network Climate Risk Note. 